point out that in an organism, left- and right-handedness is defined only with respect to the anteroposterior and dorsoventral axes. If the origins of handedness do reside in something a protein does, then the protein must be orientated with

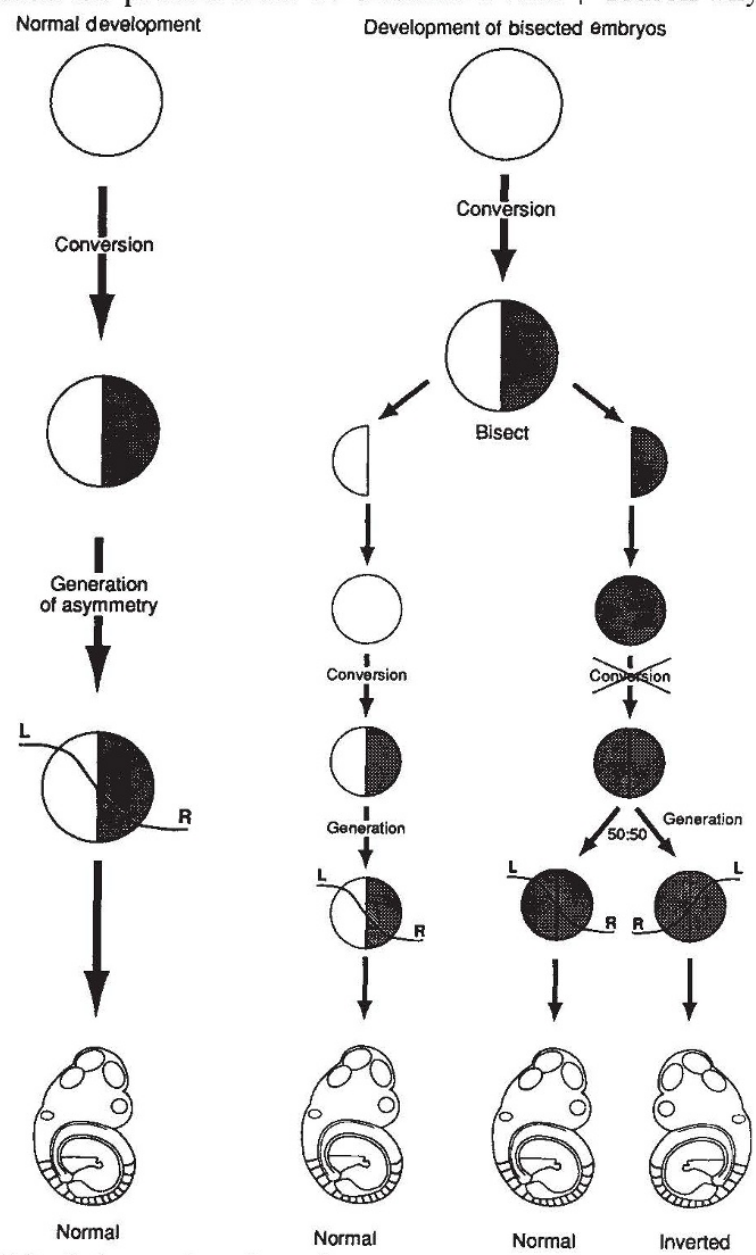

FIG. 2 An explanation of the anomalous development of hemi-embryos and conjoined twins. "Conversion" (ref. 1) is the deterministic mechanism that converts a molecular asymmetry at the cellular level and results in some stable property on the right half of an embryo. This in some way biases the otherwise random generation of asymmetry. The left half of an embryo bisected after "conversion" can develop normally, whereas the right half develops with random asymmetry because the stable property of conversion is present in both halves. (From ref. 1.)

respect to these two axes.

Orientation might well be reversible by structural changes brought about in the protein by a mutation. If the protein is a morphogenic pump, then in theory at least it could produce a consistent imbalance that biases the otherwise random part of the process. (Some of these ideas are summarized in Fig. 2) As far as it goes, Brown and Wolpert's suggestion is convincing, even if, at this stage of the game there is not much to get hold of.

How generally applicable might the model be? Certainly it could explain, as the authors believe it does, the production of situs inversus in conjoined twins of the newt Triturus (and in human twins), in which the viscera are always normal in one twin but often reversed in the other. On the other hand it does not immediately seem to offer insights into left- and righthandedness in gastropods. A recessive gene usually represents a loss of function in the normal gene product. This is one Layton's suggestion is attractwith Davis's findings ${ }^{3}$ of nongenic control of left- and right-handedness in palm trees for instance).

But for gastropods the loss of gene function does not lead to an expected and comprehensible replacement of a deterministic mechanism by a probablistic one, but by another deterministic one. Freeman and Lundelius ${ }^{10}$ found a cytoplasmic protein that will convert the abnormal left-handed screw sense of the snail Limnaea peregra to the normal right-handed one. But no equivalent protein determining lefthandedness is part of the normal development process which a separate gene can convert to right-handedness - there seems to be nothing probabilistic about the development of gastropods (or very little). In normally lefthanded gastropods like Partula the whole scheme would be reversed of course.

Whether or not the approach of Brown and Wolpert is attractive is, for now, a matter of taste. What is important is that they have suggested a philosophically sound scheme, very much in the spirit of modern embryology, of which some aspects could be tested - at least in principle. Some progress has already been made, for instance in mapping the iv gene $^{11}$, so it is on the cards that the nature of the protein will soon be known.

John Galloway is at the Cancer Research Campaign, 2 Carlton House Terrace, London SW1Y $5 A R, U K$

1. Brown, N.A. \& Wolpert, L. Development X, 1-9 (1990).

2. Boycott, A.E., Diver, C., Garstang, S.L. \& Turner, F.M. Phil. Trans. R. Soc. B219, 239-251 (1930).

3. Davis, T.A. Principes 15, 63-68 (1971).

4. Gunning, B.E.S., Hughes, J.E. \& Hardman, A.R. Planta 143, 121-144 (1978)

5. Layton, W.M. Jr. J. Hered. 67, 336-338 (1976).

6. Annett, M. Behav. Genet. 8, 227-249 (1978).

7. Driever, W. \& Nüsslein-Volhard, C. Cel/54, 83-93; 95104 (1988)

8. Kauffman, S.A., Shymko, R. \& Trabent, K. Science 199 259-270 (1978).

9. Richardson, J.S. Ad. Protein Chem. 34, 167-339 (1981).

10. Freeman, G. \& Lundelius, J.W. Wilhelm Roux Arch. dev. Biol. 191, 69-83 (1982)

11. Bruckner, A., D'Eustacco, P. \& Horwich, L. Proc. natn. Acad. Sci. U.S.A. 86, 5035-5038 (1989)

\section{Up the carbon path}

OVER half the take-off weight of a conventional space rocket is oxygen. Hence the interest in air-breathing space planes, rockets launched from aircraft to minimize first-stage weight, electromagnetic raillaunchers, even Iraqi-type super guns. Daedalus's contribution to the debate is a neat and novel air-breathing ramjet.

Its motor is simply a large block of graphite with a Venturi tube bored through it. The tube is initially filled by a charge of solid propellant. On igniting the rearward surface of this charge, it powers the vehicle into the air while heating the inner graphite surface white-hot. By the time the charge has burned away, allowing air to enter the Venturi, the craft is travelling fast enough to act as a ramjet. The air rammed into it by its forward motion burns the internal surface, and the resulting hot exhaust generates thrust.

A motor constructed entirely of fuel, of course, saves so much structural weight that it can accelerate a respectable payload to a very high final velocity. Even better, Daedalus's graphite ramjet is self-optimizing. As it rises through the atmosphere, the air pressure around it steadily falls. But burning steadily away from inside, the carbon Venturi automatically widens to capture more air, and maintains its thrust and efficiency. DREADCO's hydrodynamicists are working hard to define that initial shape of Venturi whose steady ablation by burning generates the ideal thrust envelope for space entry, and is totally consumed by the time the vehicle has risen out of the useful atmosphere. Unlike a rocket, the craft may have to climb at an angle to breathe air as long as possible.

Sooner or later, however, a rocket stage must take over to insert the craft in orbit. But rather than launching a separate second stage, Daedalus is designing an enlarged graphite ramjet which gradually turns into a rocket at the appropriate stage of the climb. Its outer layers are loaded with a graded proportion of a solid oxidizer like ammonium perchlorate. As ramaction begins to fail, combustion will reach these layers, thus compensating for the increasing oxygen deficiency. As the motor becomes a pure rocket, a multi-leaf oneway valve (almost the only moving part in the whole assembly) will seal the air inlet to prevent the propulsion gases emerging in the wrong direction.

This elegant and efficient space vehicle, lifting little deadweight and no more oxygen than it needs, should reduce satellite launching to a cheap and reliable routine. With its almost arbitrarily high fuel/payload ratio, it will speed small space probes right up to Earth-escape velocities as well, for interplanetary exploration.

David Jones 